\title{
A CRITICAL ASSESSMENT OF THE PAH HYPOTHESIS
}

\author{
B. D. DONN, \\ Code 690, Laboratory for Extraterrestrial Physics, \\ NASA/Goddard Space Flight Center, \\ Greenbelt, Maryland 20771 \\ J. E. Allen, \\ Code 691, Astrochemistry Branch, \\ NASA/Goddard Space Flight Center, \\ Greenbelt, Maryland 20771 \\ R. K. KHANNA \\ Department of Chemistry and Biochemistry, \\ University of Maryland, \\ College Park, Maryland 20742
}

\begin{abstract}
The proposal that polycyclic aromatic hydrocarbons (PAHs) are the source of the unidentified infrared bands has several serious deficiencies that have not been discussed or satisfactorily treated: (1) no collection of neutral PAHs has been found which matches the observed wavelengths; (2) ion or dehydrogenated molecules have been predicted to be the dominant species in some regions, but no infrared spectra of either species have been obtained; (3) the restriction to small grains is based on grain temperatures $\sim 1000-1500 \mathrm{~K}$ which in turn followed from the infrared continuum color temperature - there is now a question whether that is thermal emission; if it is, the source cannot be molecules; (4) recent observations of the $12 / 100 \mu \mathrm{m}$ flux ratio as a function of stellar temperature do not conform to predictions of the PAH hypotheses; (5) the photon excitation mechanism for infrared emission by neutral molecules should produce strong visible-ultraviolet fluorescence which is not observed - ions may not do this, but their infrared spectra are not known. There does not seem to be a ready explanation for these problems with the PAH hypotheses. Until definitive experimental study and analysis yielding unambiguous results have been carried out for PAHs, hydrogenated amorphous carbon or other forms of carbonaceous material, it is premature to assume any type of grain is the source of the infrared bands.
\end{abstract}

\section{INTRODUCTION}

A discussion of this nature, namely a critique of an hypothesis in an actively developing area, has inherent problems. As observations and laboratory experiments 
continue to be carried out, points of agreement and differences between predictions of the hypothesis and the observations can change rapidly. With almost each presentation concerning the unidentified infrared bands or related material, changes had to be made in this review in real time. By the time this report is published, it may be obsolete or prophetic.

The main objective of this paper is to emphasize that the proposal that the unidentified infrared bands are due to polycyclic aromatic hydrocarbons (PAHs) has not been definitely demonstrated. There are a variety of very suggestive comparisons between observation and experiment, but there are also still unexplained discrepancies and some inconsistencies. Although the PAH hypothesis has exciting potential, it is premature to claim that PAHs are abundant and ubiquitous in space.

In 1946, John Platt suggested that if one were treating the optical properties of small graphite grains, appropriate models to use are polycyclic aromatic hydrocarbons. This line of reasoning was followed in two papers (Donn, 1968; Donn and Krishna Swamy, 1969). The second paper showed that the ultraviolet absorption spectrum of a particular PAH could be significantly smoothed by combining the spectra of its derivatives and the ions (Figure 1). If similarly treated spectra of a distribution of PAHs, which was a decreasing function of the number of rings, were summed a good match to the $\lambda^{-1}$ extinction law was obtained.

These points were made well before any infrared measurements of interstellar or circumstellar regions were available. In 1973 two bands were discovered by Gillett et al. (1973) in the 8-13 $\mu \mathrm{m}$ spectrum of the planetary nebula NGC 7027. Shortly thereafter an array of seven bands was found in the 3-14 $\mu \mathrm{m}$ spectra of a variety of objects (Aitken, 1981). Electronic transitions producing the ultraviolet visible spectrum and vibrational transitions yielding the infrared features must be considered together in predicting the spectrum produced by polycyclic aromatic hydrocarbons.

\section{THE PAH HYPOTHESIS}

Sellgren and colleagues (Sellgren et al., 1983; Sellgren, 1984) reported continua in the 1-5 $\mu \mathrm{m}$ region of a number of reflection nebula that could be fitted by a black-body at a temperature of 1000-1500 K. Although the intensity decreased with distance from the exciting star, the color temperature remained constant. In order to attain temperatures in the observed range, this was explained as photon heating of small grains containing only 50-100 atoms. Léger and Puget (1984) and Allamandola et al. (1985) attributed these small refractory grains to carbon particles in the form of polycyclic aromatic hydrocarbon molecules. These species have infrared spectra generally similar to the seven unidentified bands. Thus, Léger and Puget sought to explain both the continua and emission bands by PAH molecules. Both sets of authors pointed out that the photon excitation mechanism does not produce a black-body spectrum and a more complex mechanism is required. Further analyses have called attention to the high probability that the interstellar PAHs will be both partially dehydrogenated and ionized (Allamandola et al., 1985; Léger and $\mathrm{d}$ 'Hendecourt, 1987). In addition to those included in this volume, detailed reviews of the PAH hypotheses have been given by Léger and d'Hendecourt (1987) and Allamandola et al. $(1987 a, 1987 b)$. 


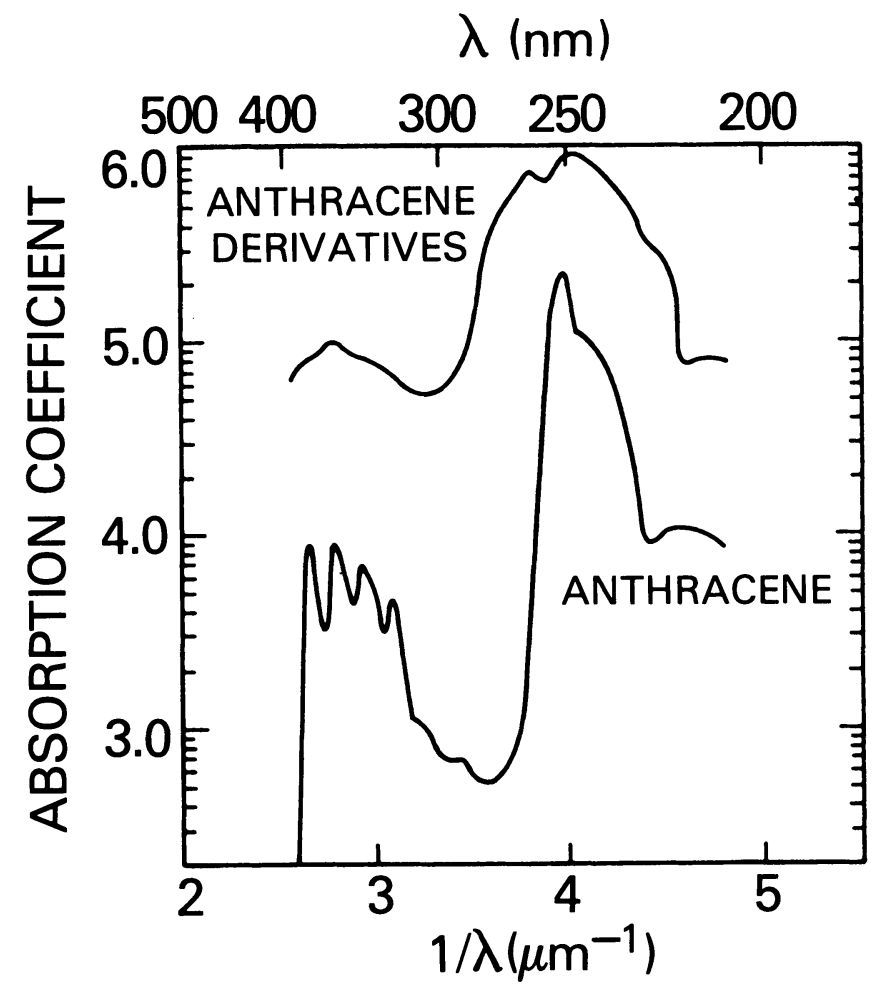

Fig. 1. Comparison of the absorption spectrum of anthracene in solution with anthracene plus thirteen derivatives or ions.

\section{PROBLEMS WITH THE PAH HYPOTHESIS}

Problems with the PAH hypothesis fall into several categories which have varying degrees of seriousness.

\subsection{SEMANTICS}

The first is semantics. There is some tendency to talk rather loosely of PAHs, hydrogenated amorphous carbon (HAC) material and carbonaceous matter as though they are all equivalent. PAHs are well-characterized chemical species with definite composition, spectral and physical properties. HACs are less well-defined substances, a characteristic of amorphous material. They are composed of hydrogen and carbon with a variable hydrogen-to-carbon ratio. Finally, carbonaceous material consists of a poorly-defined organic carbon species or mixture of species consisting of carbon, hydrogen, oxygen, nitrogen and possibly other elements in 


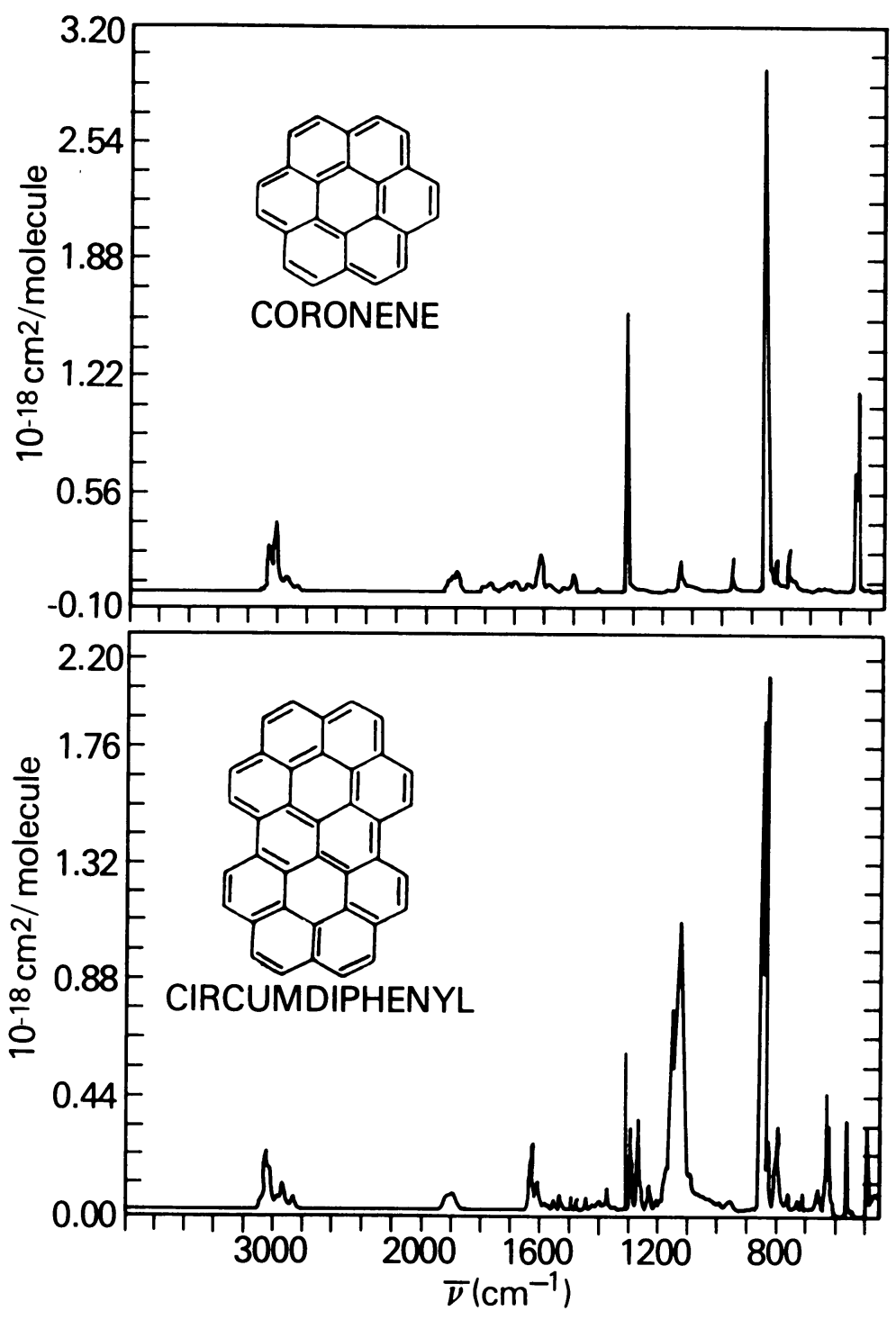

Fig. 2. (a): Room temperature absorption spectrum of coronene in $K B r$ pellet. (b) Same for circumdiphenyl. 
variable proportions. It is necessary to be careful and explicit as to what kind of material is being discussed.

\subsection{CONTINUUM}

The initial motivation for PAH molecules was to account for the approximately 1500 $\mathrm{K}$ continuum via photon excitation of small particles. As both groups of proponents recognized, molecules do not produce a black-body continuum. A combination of overtones may produce a quasi-continuum but it will not have a temperature associated with it.

At this symposium, Sellgren (1989) reported new observations suggesting that the continuum extended to longer wavelengths and was too flat to be interpreted as black-body radiation. The apparent constancy of the continuum shape with distance from the exciting star would support a photon mechanism. What these new observations bring into question is the conclusion that the source material has a relatively high temperature, $\sim 1000 \mathrm{~K}$. This conclusion was the basis for adopting small grains and PAHs.

\subsection{INFRARED EMISSION BANDS}

The primary basis for associating PAHs with the infrared emission bands is the similarity between laboratory and observational spectra (Figure 2 and 3 ). However, all comparisons, e.g., Allamandola et al. (1987a) found significant disagreements with both frequencies and intensities of the array of bands. The tendency has been to account for that problem by pointing out that spectra exist only for the neutral, fully hydrogenated molecules whereas ionized, partially dehydrogenated species are expected to occur in space. However, no infrared spectra of either ionized or dehydrogenated $\mathrm{PAH}$ molecules have yet been obtained. Therefore, no reliable comparison can be made with the observed infrared bands and no conclusions can be reached concerning the ability of PAHs to reproduce the observations. Only a few ultraviolet-visible electronic spectra for ions have been measured. These were in inert matrices, not for isolated, gas-phase molecules.

In support of the PAH hypothesis, Barker et al. (1987) attributed great significance to a series of weak bands in the $3 \mu \mathrm{m}$ region that are associated with the strong $3.3 \mu \mathrm{m}$ feature. They ascribe the bands at $2940 \mathrm{~cm}^{-1}$ to $2 \rightarrow 1$ and at $2840 \mathrm{~cm}^{-1}$ to $3 \rightarrow 2$ transition accompanying the strong $1 \rightarrow 0$ transition. Weaker features are assigned to less-abundant molecules. Such an explanation appears to require a strongly-peaked $\mathrm{PAH}$ distribution in which only a few discrete features appear rather than a broad featureless blend. However, the room temperature absorption spectra shown in Figures 2 and 3 also contain these weak features. At room temperature $\left(E \approx 200 \mathrm{~cm}^{-1}\right)$ the population of excited vibrational levels from 3000 to $9000 \mathrm{~cm}^{-1}$ is between $10^{-7}-10^{-20}$. Hence, excited levels produced by photon excitation are not necessary and there is therefore a discrepancy in the explanation.

Another difficulty is underscored by the recent observation of Sellgren et al. (1988), also discussed by Sellgren (1989). If the $12.5 \mu \mathrm{m}$ and $100 \mu \mathrm{m}$ emission bands are produced by different excitation mechanisms, their relative intensities will depend upon the temperature of the excitirg star. If the emission efficiencies do not depend upon stellar temperature and all the stellar flux contributes to the 


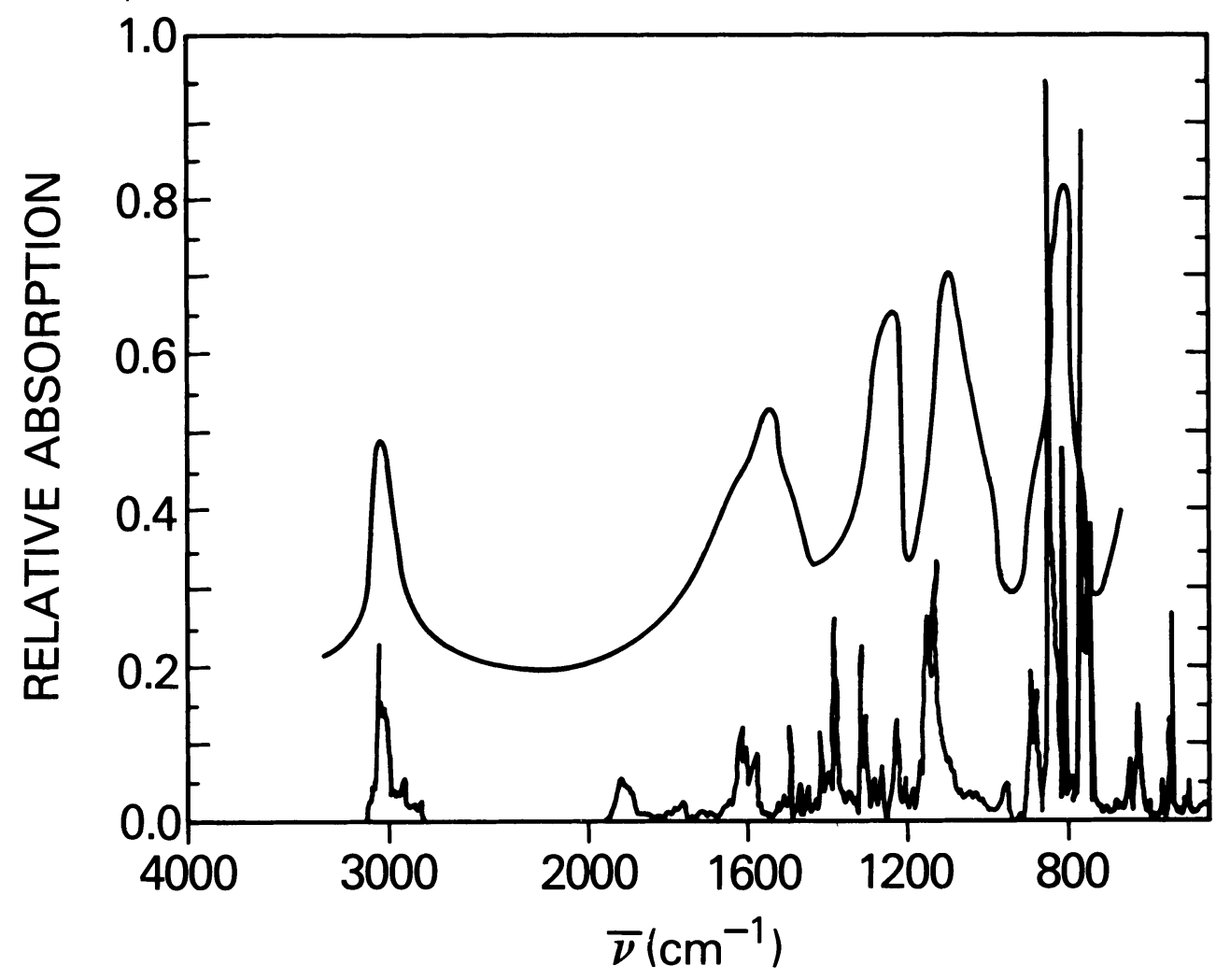

Fig. 8. Combined room temperature absorption spectra of six pericondensed PAHs in $K B r$ pellets (ovalene, coronene, circumdiphenyl, Benz-perylene, herabenzocoronene and perylene) compared with the emission spectrum of NGC 7023 (adopted from Sellgren et al., 1986).

thermal $100 \mu \mathrm{m}$ band but only the ultraviolet flux $<400 \mathrm{~nm}$ contributes to the fluorescent $12.5 \mu \mathrm{m}$ feature, then the intensity ratio $I(12.5) / I(100)$ decreases by a factor of 10 as the stellar temperature falls from $21,000 \mathrm{~K}$ to $5000 \mathrm{~K}$. Sellgren $e t$ al. (1988) found that this ratio does not seem to depend upon the stellar temperature. A possible explanation is high ultraviolet extinction in the nebulae. If this observed ratio is real, a revision of the excitation mechanism is required.

\subsection{ULTRAVIOLET-VISIBLE SPECTRUM AND FLUORESCENCE}

In the PAH hypothesis the proposed excitation mechanism is initiated by the absorption of an ultraviolet photon, leaving the molecule in an electronically and vibrationally excited state. This is followed by rapid intramolecular processes that relax the molecule to the lowest singlet or triplet state. If the molecule is initially ionized, then these lower states will be doublets or quartets. Within the PAH hypothesis only the vibrational transitions producing the infrared bands characteristic 


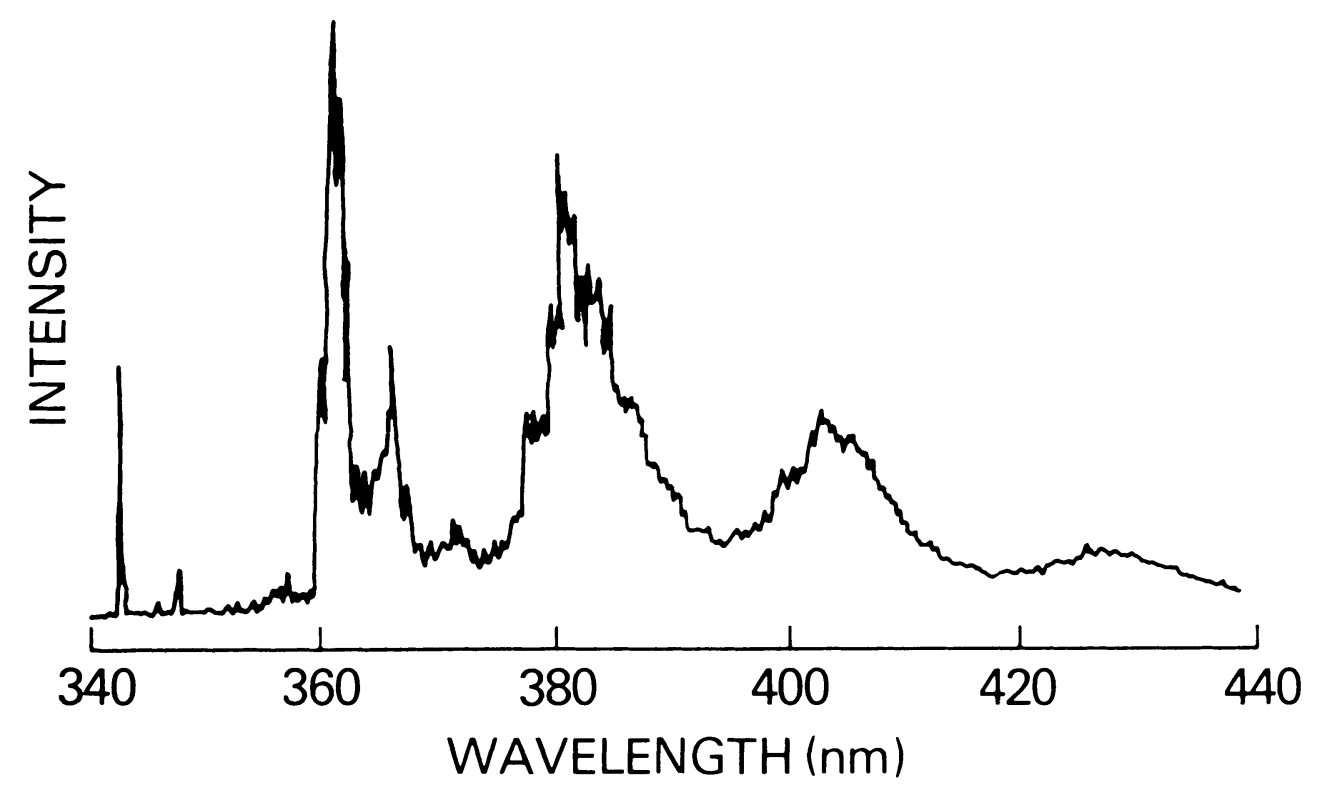

Fig. 4. Fluorescence spectrum of jet-cooled anthracene from laser excitation in $S_{1}$ electronic manifold, $E_{v i b}=1792 \mathrm{~cm}^{-1}$ (after Felker and Zewail, 1984).

of the molecule are considered. Because electronic transitions are several orders of magnitude more rapid than vibrational transitions, the ultraviolet-visible fluorescence from the first excited state is expected to be more intense than the infrared emission. d'Hendecourt et al. (1986) offer an explanation as to why fluorescence between singlet states would be weak and that red triplet-singlet phosphorescence would dominate the spectrum for large molecules. This explanation, which would overcome a crucial problem, conflicts with experimental measurements on laserexcited anthracene (Felker and Zewail, 1984) and perylene (Bouzou et al., 1983) molecules isolated in a supersonic jet. Figure 4, taken from Felker and Zewail, shows the fluorescent spectrum of jet-cooled anthracene which was initially excited by a laser to a high vibrational level of a high-lying singlet state. These results contradict the suggestion of d'Hendecourt et al. (1986) that in complex molecules ultraviolet-visible fluorescence is weak.

The absence of the ultraviolet-visible fluorescence in reflection nebula (Witt and Schild, 1988) remains as a serious objection to the PAH hypothesis. One may try to circumvent this non-observance of the UV-VIS fluorescence by proposing that a complex mixture of polycyclic aromatic molecules washes out any structure or strong blue features. This was originally suggested to occur in the extinction proposal, shown in Figure 1. In this case, the infrared spectrum would be expected to lose its simple structure, which diminishes the suggestive agreement with the observations. Another possible explanation for the absence of fluorescent features 
would be the ionization of the molecules. Leach's (1987) discussion of polycyclic aromatic ions indicates that the energy gap between the ground and first excited electronic state correspond to red or near-infrared emissions. This explanation is also expected to cause significant disagreement in the infrared although, as pointed out, no infrared spectra are yet available for ions.

\section{ALTERNATIVE EXPLANATIONS FOR THE INFRARED BANDS}

There can be little question that the unidentified infrared bands have their origin in some form of hydrogenated carbonaceous material. The similarity of the band positions with hydrocarbon material first pointed out by Duley and Williams (1981) is too convincing to overlook. However, polycyclic aromatic hydrocarbons are not the only possible explanation. Allamandola et al. (1987a) stated "Thus, on the basis of spectroscopic evidence alone, we would conclude that amorphous carbon particles are responsible." They went on to say that intensity characteristics require infrared fluorescence from molecular-sized species. The array of problems described here was not treated.

The nominal room temperature condensate of a discharge in methane (Sakata et al., 1984), called quenched carbonaceous composite (QCC), has bands at 3.29, 3.42, $3.48,6.52,6.94,7.27,11.40,11.96$ and $13.24 \mu \mathrm{m}$. Heating causes intensity changes among the bands and gradual disappearance of most bands. When oxidized (Sakata et al., 1987) the deposit shows additional bands at 7.7 and $8.6 \mu \mathrm{m}$. Borghesi et al. (1987) have produced amorphous carbon grains by striking an arc between carbon electrodes in argon. The infrared spectra of these grains showed bands at 3.4, 3.51, $5.787,6.29,6.85$, and $11.3 \mu \mathrm{m}$. Some of these bands also show a strong temperature dependence. Duley and Williams at this symposium (Duley and Williams, 1989) proposed another structure for interstellar grains which may also account for the infrared bands.

It is necessary to experimentally pursue all the mechanisms discussed here as well as any new or modified proposals. The question of the explanation of the unidentified infrared bands at this time is still open. It is a mistake to adopt any one proposal as the answer before a definite study has been performed.

ACKNOWLEDGEMENTS. We are grateful to David Salisbury for providing Figures 2 and 3.

\section{REFERENCES}

Aitken, D. K. 1981, in IAU Symposium, 96, Infrared Astronomy, eds. C. G. Wynn-Williams and D. P. Cruickshank, (Boston: Reidel), p. 207.

Allamandola, L. J., Tielens, A. G. G. M. and Barker, J. R. 1985, Ap. J. (Letters), 290, L25. . 1987a, in Polycyclic Aromatic Hydrocarbons and Astrophysics, eds. A. Léger, L. B. d'Hendecourt, and N. Boccara, (Boston: Reidel), p. 255.

- 1987b, in Physical Processes in Interstellar Clouds, eds. G. E. Morfell and M. Scholer, (Boston: Reidel), p. 305.

Barker, J. R., Allamandola, L. J. and Tielens, A. G. G. M. 1987, Ap. J. (Letters), 815, L61.

Borghesi, A., Bussoletti, E. and Colangeli, L. 1987, Ap. J., \$14, 422. 
Bouzou, C., Jouvet, C, LeBlond, J. B., Miller, Ph. and Tramer, A. 1983, Chem. Phys. Letters, 97, 2.

Donn, B. 1968, Ap. J., 152, L129.

Donn, B. and Krishna Swamy, K. S. 1969, Physica, 41, 133.

Duley, W. and Williams, D. A. 1981, M. N. R.A.S., 196, 269. 1989, in Interstellar Dust Contributed Papers, eds. A. G. G. M. Tielens and L. J. Allamandola, NASA CP-3036.

Felker, P. M. and Zewail, A. H. 1984, Chem. Phys. Letters, 108, 303.

Gillett, F. C., Forrest, W. J. and Merrill, K. M. 1973, Ap. J., 183, 87.

d'Hendecourt, L. B., Léger, A., Olofsson, G. and Schmidt, W. 1986, Astr. Ap., 170, 91.

Leach, S. 1987, in Polycyclic Aromatic Hydrocarbons and Astrophysics, eds. A. Léger, L. d'Hendecourt and N. Boccara (Boston: Reidel), p. 99.

Léger, A. and Puget, J. L. 1984, Astr. Ap., 137, L5.

Léger, A. and d'Hendecourt, L. B. 1987, in Polycyclic Aromatic Hydrocarbons and Astrophysics, eds. A. Léger, L. d'Hendecourt and N. Boccara (Boston: Reidel), p. 223.

Sakata, A., Wada, S, Tanabe, T. and Onaka, T. 1984, Ap. J. (Letters), 287, L51.

Sakata, A., Wada, S., Onaka, T. and Tokunaga, A. T. 1987, Ap. J. (Letters), 320, L63.

Sellgren, K. 1984, Ap. J., $277,623$.

Sellgren, K., Werner, M. W. and Dinerstein, H. L. 1983, Ap. J., 271, L13.

Sellgren, K., Allamandola, L. J., Bregman, J. D., Werner, M. W. and Wooden, D. H. 1986, Ap. J., $299,416$.

Sellgren, K., Castelaz, M. W., Werner, M. W. and L. Lunan 1988, in Comets and Cosmology, ed. A. Lawrence, (New York: Springer Verlag).

Sellgren, K. 1989, in IAU Symposium 195, Interstellar Dust, eds. L. J. Allamandola and A. G. G. M. Tielens, (Dordrecht: Kluwer), p. 103.

Witt, A. N. and Schild, R. E. 1988, Ap. J., \$25, 837. 